\title{
Fatores Associados a Tensão Arterial Não Controlada em Pessoas com Diabetes Tipo 2 Seguidas nos Cuidados de Saúde Primários em Portugal
}

\author{
Characteristics Associated with Uncontrolled Blood \\ Pressure Among Portuguese Primary Care Patients with \\ Type 2 Diabetes
}

Inês ROSENDO $\triangle^{1,2}$, Luíz Miguel SANTIAGO ${ }^{3,4}$, Margarida MARQUES ${ }^{5}$

Acta Med Port 2017 Mar;30(3):197-204 - https://doi.org/10.20344/amp.8321

RESUMO

Introdução: A hipertensão arterial está associada a maior morbimortalidade na diabetes tipo 2. É assim importante identificar quais as características socio demográficas, hábitos e fatores de risco das pessoas com diabetes tipo 2 que estão associadas a descontrolo tensional de modo a intervir de forma ajustada nesta população.

Material e Métodos: Estudo observacional transversal nos cuidados de saúde primários com recolha de dados de pessoas com diabetes tipo 2 aleatorizadas pelos seus médicos de família, estratificados proporcionalmente pelas cinco regiões de saúde de Portugal continental. Variáveis: tensão arterial, idade, sexo, formação, duração da diabetes, HbA1c, hábitos tabágicos, peso, perímetro abdominal, nível de atividade física e adesão à medicação. Análise bivariada e por regressão logística para avaliar a associação independente de cada variável com descontrolo tensional (tensão arterial $\geq 140 / 90$ ).

Resultados: Estudados 709 doentes, 60,2\% homens, idade média 66,12 $\pm 10,47$ anos. Após análise de regressão logística, verificouse que os fatores independentemente associados a não controlo da tensão arterial foram a menor formação académica $(p=0,014)$, a menor duração da diabetes $(p=0,002)$, o maior perímetro abdominal $(p<0,001)$, a maior pressão de pulso $(p<0,001)$ e o maior nível de atividade física $(p=0,043)$, assim como o facto de ser fumador $(p<0,001)$.

Discussão: As principais limitações do estudo são o facto da amostra não ser totalmente aleatória e incluir apenas frequentadores dos cuidados primários, um possível vies inter-observador e o facto de ser um estudo transversal e não poder deduzir causalidade. Conclusão: O sub-grupo de pessoas com diabetes tipo 2 identificadas como tendo maior associação a pior controlo tensional deveriam ser alvo de uma abordagem diferente e mais intensiva nos cuidados de saúde primários em relação a este fator de risco. Recomendamos estudos longitudinais e populacionais para confirmar estes resultados.

Palavras-chave: Cuidados de Saúde Primários; Determinação da Pressão Arterial; Diabetes Mellitus Tipo 2; Portugal; Pressão Arterial

\section{ABSTRACT}

Introduction: Determine whether socio-demographic, habits and risk factors are associated with a better tensional control in type 2 diabetes in primary care patients in order to identify a specific target population for compensatory interventions improving diabetes control and reducing its morbi-mortality.

Material and Methods: Cross-sectional study in primary care. Randomized type 2 diabetes patient data collection by their volunteer family doctors, proportionally stratified from the 5 Portuguese continental regions. Variables: blood pressure, age, gender, education, diabetes duration, $\mathrm{HbA} 1 \mathrm{c}$, smoking habits, weight, waist circumference, physical activity and adherence to medication. Bivariate and logistic regression analysis to evaluate each measured variable's independent association with uncontrolled blood pressure ( $\geq 140 / 90)$. Results: 709 patients were included in the study, 60.2\% men, mean age $66.12 \pm 10.47$ years. In logistic regression analysis, the factors independently associated to uncontrolled BP were lower education $(p=0.014)$, shorter diabetes duration $(p=0.002)$, higher waist circumference $(p<0.001)$, higher pulse pressure $(p<0.001)$, higher physical activity level $(p=0.043)$ and being a smoker $(p<0.001)$. Discussion: The main limitations are the fact that the sample was not totaly random and included only primary care patients, a possible inter-observer bias and being a cross-sectional study, thus not providing information on temporal relation or causality.

Conclusion: The sub-group of people with diabetes identified to have worse tensional control should have a different and more intensive approach in primary care. We recommend further longitudinal and population based confirmatory research.

Keywords: Blood Pressure; Blood Pressure Determination; Diabetes Mellitus, Type 2; Portugal; Primary Health Care

\section{INTRODUÇÃO}

A diabetes mellitus (DM) sendo um problema de Saúde Pública de elevada magnitude, constitui já uma das principais causas de morbilidade e incapacidade total ou parcial durante o século XXI. Em 2015, estima-se a existência de 415 milhões de pessoas com diabetes no mundo e que em
2040 este valor ascenda a 642 milhões. Em 2015, a diabetes provocou cinco milhões de mortes a nível mundial. ${ }^{1}$

A prevalência da diabetes está a aumentar. Em Portugal, segundo o Relatório Anual do Observatório Nacional da Diabetes, no ano de 2014, na população dos 20 aos

1. Unidade de Cuidados de Saúde Primários Fernão de Magalhães. Coimbra. Portugal.

2. Faculdade de Medicina. Universidade de Coimbra. Coimbra. Portugal.

3. Unidade de Saúde Familiar Topázio. Coimbra. Portugal.

4. Faculdade de Ciências da Saúde. Universidade da Beira Interior. Covilhã. Portugal.

5. Laboratório de Bioestatística e Informática Médica. Faculdade de Medicina. Universidade de Coimbra. Coimbra. Portugal.

$\triangle$ Autor correspondente: Inês Rosendo. inesrcs@gmail.com

Recebido: 17 de outubro de 2016 - Aceite: 07 de fevereiro de 2017 | Copyright @ Ordem dos Médicos 2017 
79 anos a prevalência da diabetes estimada era de 13,3\%, tendo crescido 1,4 pontos percentuais desde 2009. Em $2014,4 \%$ das mortes tiveram como origem esta doença e em 2013, a diabetes representou cerca de oito anos de vida perdida por cada óbito por diabetes na população com idade $<70$ anos. $^{2}$

Esta doença é responsável por uma elevada frequência de consultas e de atendimentos de urgência, assim como por um grande número de internamentos hospitalares. A despesa com medicamentos para a diabetes tem aumentado, tendo correspondido a uma despesa para o SNS de 43,1 milhões de euros em 2014 e as despesas totais com a diabetes em Portugal em 2014 representaram 0,7\% - 0,9\% do PIB e $8 \%$ - $10 \%$ da despesa em saúde. ${ }^{2}$

A principal causa de morbimortalidade e custos diretos e indiretos nas pessoas com diabetes é a doença cardiovascular aterosclerótica. As patologias que normalmente coexistem com a diabetes (como a hipertensão e dislipidémia) são fatores de risco cardio-vascular (RCV) e a própria diabetes confere risco de forma independente. ${ }^{3}$ Em Portugal, confirmou-se também a forte associação da DM à doença coronária (DC) e ao acidente vascular cerebral (AVC). ${ }^{4} \mathrm{Nu}-$ merosos estudos mostram a eficácia do controlo individual de cada um desses fatores de risco e foram demonstrados grandes benefícios quando são controlados vários fatores simultaneamente. ${ }^{5}$ Está recomendado avaliar-se sistematicamente os fatores de RCV pelo menos anualmente em todas as pessoas com diabetes: hipertensão, dislipidémia, hábitos tabágicos, microalbuminúria e história familiar de doença coronária precoce.

A hipertensão (HTA) é um fator de risco major para doença cardiovascular aterosclerótica e complicações microvasculares e é mais prevalente nas pessoas com diabetes. ${ }^{6}$ Em Portugal, a associação entre DM e HTA parece ser considerável, com $78,3 \%$ das pessoas com diabetes consideradas hipertensas. ${ }^{7}$

Nesta população, há evidência de que o controlo adequado dos valores de TA diminui a mortalidade, risco de eventos cardiovasculares, albuminúria e retinopatia. ${ }^{8-10}$ Foi determinado um NNT (número de pessoas necessário tratar) de seis para prevenir uma complicação major da diabetes a 10 anos e de 15 para prevenir morte a 10 anos,,$^{11}$ sendo até considerado mais importante do que o controlo glicémico pois a intervenção intensiva com esse fim não conseguiu provar redução nestes endpoints. ${ }^{12,13}$ Esta intervenção de controlo da TA provou ser também custo-efetivo. ${ }^{14}$ Há forte evidência de que valores de TA sistólica superiores a $140 \mathrm{~mm} \mathrm{Hg}$ e TA diastólica superiores a $90 \mathrm{mmHg}$ são perigosos mas baixar muito abaixo deste valor não parece ter benefício. ${ }^{15}$

Foram associadas a melhor controlo da hipertensão várias características socio-demográficas dos doentes como o sexo masculino, ${ }^{16,17}$ a idade mais jovem ${ }^{17-19}$ e maior formação. ${ }^{19,20}$ Quanto a variáveis associadas a outros fatores de risco, o controlo tensional foi associado a menores níveis de colesterol LDL, ${ }^{18,21}$ menor IMC, ${ }^{18,19,21}$ maior nível de atividade física ${ }^{22}$ e maior duração da diabetes. ${ }^{18,21}$
Em Portugal, em 2006/2007 apenas 37,9\% dos utentes com diabetes tinham TA $<140 / 90^{7}$ e em 2014 , este número era já de $66,7 \%$, nos registos dos cuidados de saúde primários. $^{2}$

Sabendo quais as características das pessoas com diabetes associadas a pior controlo da tensão arterial, poderemos intervir nesses sub-grupos de forma diferenciada de forma a estrategicamente investir na redução da morbimortalidade associada a esta doença. Não existe nenhum estudo publicado visando perceber a influência de características socio-demográficas, hábitos e fatores de risco no controlo da tensão arterial em pessoas com diabetes em Portugal e por isso este foi o objetivo do presente estudo.

\section{MATERIAL E MÉTODOS}

Estudo transversal, observacional em amostra constituída pelos 18 primeiros doentes com diabetes tipo 2 que recorreram a consulta de seguimento pelo seu Médico de Família a partir de 15 de outubro de 2014. Foi feito o convite nas redes sociais a médicos de família e aceites voluntários até atingir 65 médicos de Portugal continental, distribuídos pelas cinco ARS (25 no Norte, 11 no Centro, 23 em Lisboa e Vale do Tejo, três no Alentejo e três no Algarve). O número de médicos em cada região foi determinado por amostragem multi-etápica após estratificação, partindo da geodemografia de cada região (dados do INE de 2011 e do estudo PREVDIAB de $2009^{23}$ sobre número de doentes com diabetes e população por região) e fazendo uma distribuição tendencialmente proporcional dos casos pelas regiões (para obter o número total calculado como necessário), agrupando depois estes casos por número de médicos de família a recrutar (18 doentes com diabetes/ médico). Foram excluídos doentes menores de 18 anos, grávidas, acamados, pessoas em cadeira de rodas ou com diagnóstico de depressão na lista de problemas.

As variáveis estudadas foram: sexo, idade, formação académica (número de anos de escolaridade), tempo de evolução da diabetes (anos), HbA1c (feita em ambulatório e trazida à consulta ou medida no centro de saúde no dia da consulta) e o facto de ser insulinotratado (sim/não). Foi ainda medido na consulta o peso e índice de massa corporal (IMC), perímetro abdominal (PA) - recomendado medir no ponto médio entre a crista ilíaca e a margem costal inferior $^{24}$ - e tensão arterial (TA) - recomendada medir pelo menos duas vezes na posição sentada, registando a média. ${ }^{25}$ Foram consideradas controladas a nível da TA, as pessoas que apresentavam nesta medição feita na consulta uma TA $<140 / 90$. Foram inquiridos os hábitos tabágicos (número de cigarros/dia), a actividade física praticada (instrumento PACE em Português) ${ }^{26}$ e adesão/manutenção em terapêutica (escala Morisky Medication Adherence Scale em Português). ${ }^{27}$ Foram registados os medicamentos anti-diabéticos orais e anti-hipertensores prescritos.

$\mathrm{Na}$ estatística descritiva os valores qualitativos são apresentados com $n^{\circ}$ e $\%$ e os quantitativos com média \pm desvio padrão. Na estatística inferencial recorreu-se à análise de qui-quadrado e ao teste $U$ de Mann Witney para 
comparar respetivamente variáveis qualitativas e quantitativas entre doentes com a TA controlada e não controlada. Foi ainda feita uma análise multivariada com a regressão logística (método backward incluindo os fatores com nível de significância $<0,5$ na análise bivariada) para estudar a influência de cada um destes fatores, usando análise por intenção de tratar, e calculando odds ratio. Todos os cálculos e testes de associação entre variáveis ou grupos de indivíduos foram feitos para um nível de significância de 0,05 .

\section{RESULTADOS}

Nesta amostra, composta por 709 doentes recrutados por 41 médicos de família a nível nacional (63\% dos recrutados inicialmente), $60,2 \%$ dos doentes eram do sexo masculino, tinham em média 66,12 $\pm 10,47$ anos e 6,26 $\pm 3,90$ anos de formação, sendo 1,7\% analfabetos e 9,3\% tendo formação superior ao $12^{\circ}$ ano. Em média, tinham diabetes há $9,25 \pm 7,83$ anos e $13 \%$ eram insulino-tratados.

A TA sistólica média era de 137,02 \pm 16,45 mm Hg e diastólica 76,48 \pm 10,65 mm Hg (Tabela 1), tendo 57,1\% dos doentes a TA sistólica controlada e $88,3 \%$ a TA diastólica controlada. A pressão de pulso média era de 60,54 $\pm 15,34 \mathrm{mmHg}$. Na amostra, $54,6 \%$ dos doentes tinham a $\mathrm{TA}<140 / 90$. Estavam medicados com pelo menos um anti-hipertensor $83,1 \%$ dos doentes estando não medicados $9,9 \%$ dos que não estavam controlados.

Quanto a medidas antropométricas, o IMC médio da amostra era de $29,39 \pm 4,87$, sendo que $45,1 \%$ tinham excesso de peso, $26,2 \%$ obesidade ligeira e $11,8 \%$ obesidade severa. O PA médio era de $102,57 \pm 11,58$ e estava elevado em $88,3 \%$ das mulheres e $72,1 \%$ dos homens, e muito elevado em $81,2 \%$ das mulheres e $48,7 \%$ dos homens.

Quanto à relação entre o controlo da tensão arterial (TA < 140/90) e outras variáveis, encontrou-se associação em análise bivariada (Tabela 2) com maior formação académica $(p<0,001)$, menor IMC $(p=0,001)$, perímetro abdominal menor $(p<0,001)$, pressão de pulso menor $(p<0,001)$. Apesar de haver tendência a maior controlo nas mulheres, nos não fumadores, nos com a diabetes controlada, nos Tabela 1 - Caracterização socio-demográfica, $\mathrm{HbA} 1 \mathrm{c}$ e fatores de risco dos doentes com diabetes da amostra

\begin{tabular}{lcccc}
\hline Variável & Média \pm desvio padrão & Mínimo & Máximo & $\mathbf{n}$ \\
\hline Idade & $66 \pm 10$ & 30 & 91 & 707 \\
Formação & $6 \pm 4$ & 0 & 22 & 689 \\
Tempo evolução diabetes & $9 \pm 8$ & 0 & 47 & 12,1 \\
HbA1c & $6,8 \pm 1,0$ & 4,4 & 195,00 & 708 \\
Tensão arterial sistólica & $137 \pm 17$ & 90,00 & 120,00 & 709 \\
Tensão arterial diastólica & $77 \pm 11$ & 40,00 & 50,95 & 709 \\
Índice de massa corporal & $29,4 \pm 4,9$ & 17,62 & 113 & 709 \\
Pressão de pulso & $61 \pm 15$ & 23 & 60 & 149 \\
Perímetro abdominal & $102,6 \pm 11,6$ & & 630 \\
\hline
\end{tabular}

$\mathrm{n}=$ Número de pessoas com valor registado na variável

Tabela 2 - Fatores socio-demográficos, hábitos e fatores de risco e sua associação a controlo da tensão arterial (TA)

\begin{tabular}{|c|c|c|c|c|c|}
\hline \multicolumn{2}{|l|}{ Variável } & \multirow{2}{*}{$\begin{array}{c}\begin{array}{c}\text { Pessoas com } \\
\text { controlo TA }\end{array} \\
51,6\end{array}$} & \multirow{2}{*}{$\begin{array}{c}\begin{array}{c}\text { Pessoas sem } \\
\text { controlo TA }\end{array} \\
48,4\end{array}$} & \multirow{2}{*}{$\begin{array}{c}n \\
709\end{array}$} & \multirow{2}{*}{$\begin{array}{c}p \\
0,055\end{array}$} \\
\hline $70 m$ d & Norte/ Centro & & & & \\
\hline Zona do pals & Lisboa e Vale do Tejo/ Alentejo/ Algarve & 58,8 & 41,2 & & \\
\hline \multirow{2}{*}{ Sexo* } & Feminino & 55,3 & 44,7 & 709 & 0,749 \\
\hline & Masculino & 54,1 & 45,9 & & \\
\hline Formação** & & $6,88 \pm 4,18$ & $5,52 \pm 3,39$ & 689 & $<0,001$ \\
\hline \multirow[t]{2}{*}{ Atividade física* } & Faz & 53,4 & 46,6 & 709 & 0,310 \\
\hline & Não faz & 57,6 & 42,4 & & \\
\hline \multirow[t]{2}{*}{ Fumador* } & Sim & 50,7 & 49,3 & 709 & 0,471 \\
\hline & Não & 55 & 45 & & \\
\hline \multicolumn{2}{|c|}{ Índice de massa corporal** } & $28,85 \pm 4,74$ & $30,03 \pm 4,95$ & 708 & 0,001 \\
\hline \multicolumn{2}{|c|}{ Perímetro abdominal ${ }^{* *}$} & $101,08 \pm 11,43$ & $104,40 \pm 11,52$ & 630 & $<0,001$ \\
\hline \multicolumn{2}{|c|}{ Pressão de pulso** } & $53,25 \pm 10,32$ & $69,29 \pm 15,81$ & 709 & $<0,001$ \\
\hline \multirow[t]{2}{*}{$\mathrm{HbA} 1 \mathrm{c}^{*}$} & Controlada $(<7 \%)$ & 55,2 & 44,8 & 709 & 0,675 \\
\hline & Não controlada & 53,5 & 46,5 & & \\
\hline \multicolumn{2}{|c|}{ Duração da diabetes** } & $9,53 \pm 7,97$ & $8,91 \pm 7,65$ & 709 & 0,273 \\
\hline
\end{tabular}

$\mathrm{n}=$ Número de pessoas com valor registado na variável

* Percentagens, teste Qui-quadrado; ** Média \pm desvio padrão, teste U de Mann-Whitney. 
Tabela 3 - Fatores associados a não controlo tensional após análise de regressão logística, com risco relativo (odds ratio e intervalo de confiança (IC))

\begin{tabular}{lccc}
\hline Variável & Odds ratio & IC (95\%) & $\boldsymbol{p}$ \\
\hline Formação & 0,933 & $0,883-0,986$ & $\mathbf{0 , 0 1 4}$ \\
Fumador & 2,128 & $1,133-3,996$ & $\mathbf{0 , 0 1 9}$ \\
Atividade física & 1,136 & $1,004-1,285$ & $\mathbf{0 , 0 4 3}$ \\
Perímetro abdominal & 1,043 & $1,024-1,063$ & $<\mathbf{0 , 0 0 1}$ \\
Pressão de pulso & 1,114 & $1,093-1,126$ & $<\mathbf{0 , 0 0 1}$ \\
Duração da diabetes & 0,960 & $0,936-0,986$ & $\mathbf{0 , 0 0 2}$ \\
\hline
\end{tabular}

com maior duração da diabetes, nos pertencentes à zona sul do país e nos mais ativos, não existiu diferença estatística nestas variáveis.

Após análise de regressão logística (Tabela 3 e Fig. 1), verificou-se que os fatores independentemente associados a descontrolo da TA foram a menor formação académica e duração da diabetes e maior perímetro abdominal, pressão de pulso e nível de atividade física, assim como ser fumador. Estes fatores explicam $43,9 \%$ da variação do modelo.

\section{DISCUSSÃO}

Em termos de controlo tensional na amostra, 54,6\% dos doentes tinham a TA controlada (TA < 140/90), valor inferior ao $72,1 \%$ de controlo na população diabética seguida nos cuidados de saúde primários com registos em 2014 . $^{2}$ A diferença pode existir por apenas $80,1 \%$ terem registos a nível nacional no estudo do Observatório Português, ${ }^{2}$ em contraste com os $100 \%$ da nossa amostra, pois neste caso os dados foram recolhidos diretamente pelos médicos e não extraídos retrospetivamente dos ficheiros. Na Europa, no estudo PANORAMA e no estudo GUIDANCE, apenas $19,7 \%$ e $19,3 \%$, respetivamente, tinham a TA controlada, ${ }^{28,29}$ e nos EUA em 2009/2010 o controlo era de 52,8\% (Wong et al, 2013), mas nesses estudos foram considerados como controlo valores de TA $<130 / 80 \mathrm{~mm} \mathrm{Hg}$. Em estudos mais recentes, usando TA $<140 / 90$ como referência, verifica-se num estudo na Alemanha controlo de $36,4 \%$ dos doentes com diabetes, ${ }^{31}$ num outro na França controlo de 51,2 - 65,4\%22 e na Austrália, 45,3\%,32 mais próximos dos valores que também obtivemos. A TA sistólica apresentou menor controlo que a TA diastólica, como tem sido encontrado nos outros estudos. A maior dificuldade na obtenção de adequado controlo tensional na população diabética comparativamente à restante população, particularmente no que respeita à pressão arterial sistólica, tem sido atribuída ao efeito dos fatores de risco associados na limitação da resposta à terapêutica anti-hipertensora.

Estavam medicados com pelo menos um anti-hipertensor, $83,1 \%$ dos doentes desta amostra, número superior ao estudo VALSIM $(71,3 \%)$ nos cuidados de saúde primários em Portugal. ${ }^{7} \mathrm{O}$ valor agora obtido é superior também a um estudo populacional na Alemanha $(69,7 \%)^{31}$ mas inferior a um estudo espanhol em que estavam medicados $94,7 \%$ das pessoas com diabetes, mas que foi conduzido nos cuidados de saúde terciários. ${ }^{21}$ No nosso estudo, 9,9\% das pessoas com diabetes que não tinham a TA controlada não estavam medicados, o que sugere que a maioria necessitaria de um reforço terapêutico e não de início de tratamento, o que é consistente com outros estudos que referem uma fraca intensificação da terapêutica nesta área. ${ }^{33,34}$

Neste estudo tivemos como objetivo identificar características associadas a descontrolo da tensão arterial em doentes com diabetes. Após análise de regressão logística, verificou-se que os fatores independentemente associados

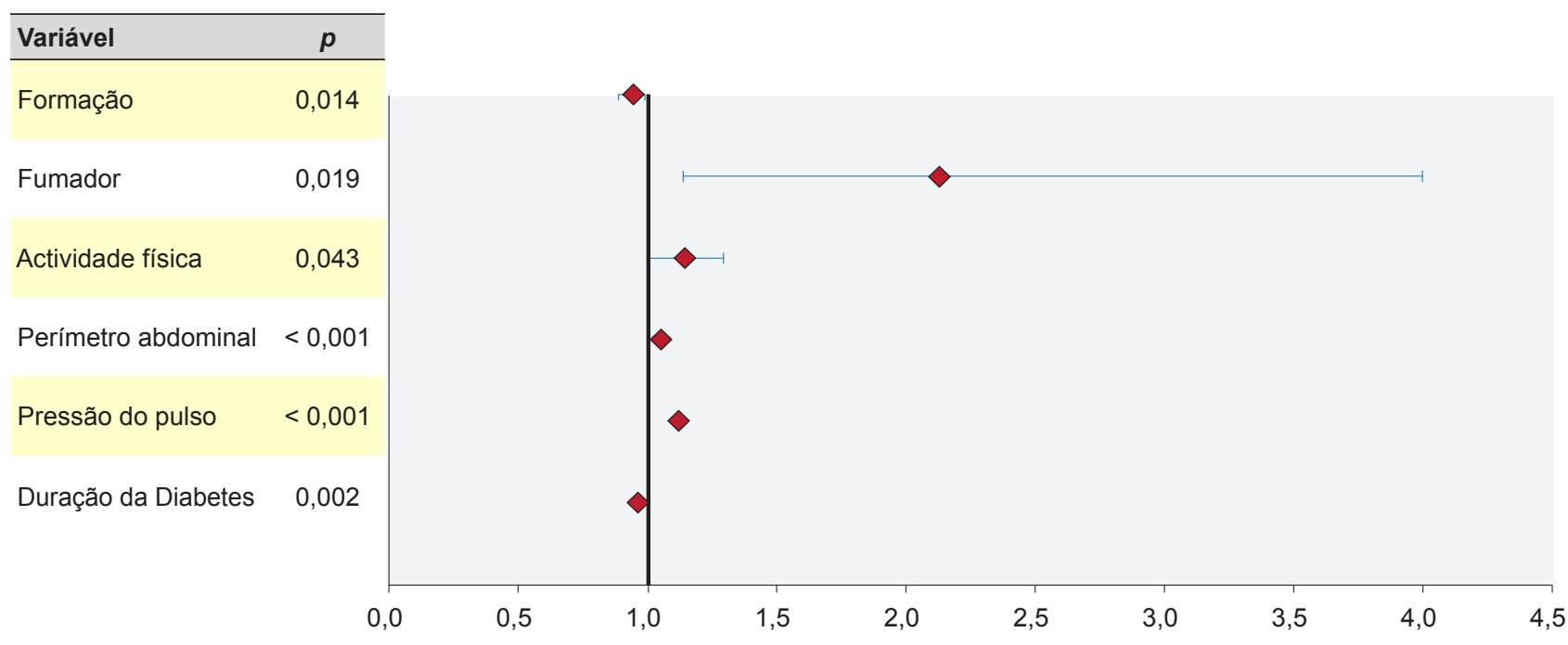

Figura 1 - Gráfico do odds ratio (vermelho) e intervalo de confiança (azul) dos fatores associados a não controlo tensional, após análise de regressão logística 
a não controlo da TA foram a menor formação académica e menor duração da diabetes e maior perímetro abdominal, maior pressão de pulso e maior nível de atividade física, assim como o facto de ser fumador.

O sexo feminino apresentou, no nosso estudo, um maior controlo, não significativo, da TA, o que está de acordo com alguns estudos. ${ }^{35,36}$ Isto pode-se explicar pela menor utilização pelos homens dos cuidados de saúde e sua menor valorização destes cuidados e de estilos de vida saudáveis. ${ }^{37,38}$

A formação académica esteve associada a maior controlo da TA, tal como noutros estudos ${ }^{19,20}$ realizados em locais com níveis globais de baixo nível de formação e socio-económica. Isto está de acordo com os estudos que referem haver um baixo nível geral de literacia em Portugal. ${ }^{39,40}$ Também há estudos que relacionam a formação académica com o maior controlo da $\mathrm{HbA1c},{ }^{41,42}$ sugerindo que a literacia em saúde influenciará os resultados da diabetes. $^{43}$

A duração da diabetes estava independentemente associada a melhor controlo da TA, o que tem sido verificado em outros estudos ${ }^{18,21}$ e que pode ser explicado por uma maior capacitação para a gestão dos fatores de risco por esses doentes. O oposto acontece com o controlo glicémico, ${ }^{28,29,44-50}$ provavelmente pela natureza progressiva da diabetes que leva a maior descontrolo glicémico com o avançar da doença.

A obesidade, apesar de associada a menor controlo tensional na análise bivariada, não estava associada de forma independente ao controlo tensional após eliminados os fatores confundentes, provavelmente pela sua relação próxima com os valores de PA. Outros estudos apresentaram resultados contraditórios, associando ao controlo tensional um maior IMC ${ }^{35}$ ou menor IMC. ${ }^{18,21}$ De facto, os doentes com mais do que um fator de risco cardiovascular além da hipertensão (pessoas com diabetes e obesos simultaneamente) parecem estar mais alerta para a sua hipertensão e estarem mais medicados mas, ao mesmo tempo, são de mais difícil obtenção de resultados apesar de tratamento, o que pode levar a estes resultados díspares. ${ }^{35}$ Já o perímetro abdominal mostrou associação independente e inversa com o controlo tensional $(p<0,001)$. Esta parece ser a variável mais preditora de síndrome metabólico nas pessoas com diabetes portuguesas, mesmo sem a presença de obesidade pelo $\mathrm{IMC}^{4}$ e corresponde a risco aumentado de mortalidade cardio-vascular associada, particularmente nos homens. ${ }^{51,52}$ Parece haver uma associação do aumento do perímetro abdominal (obesidade abdominal) às doenças cardiovasculares mais forte do que a própria obesidade, segundo o IMC. ${ }^{53} \mathrm{O}$ facto de o IMC estar associado a uma TA mais elevada no nosso estudo põe em evidência o maior risco cardiovascular desta sub-população e enfatiza a importância da valorização da obesidade abdominal nas pessoas com diabetes.

A pressão de pulso esteve também associada de forma independente a valores mais elevados de TA. Esta parece estar associada a mais complicações micro e macrovascu- lares em doentes com diabetes ${ }^{54}$ e mais uma vez chama à atenção para a importância de valorizar os valores da pressão de pulso em pessoas com diabetes.

A atividade física esteve independentemente relacionada com pior controlo tensional. Este fator tem sido pouco estudado como preditor de controlo tensional mas sabe-se que a atividade física tem efeitos benéficos na diminuição da TA em pessoas com diabetes..$^{22,55-57}$ Ainda assim, há diversos estudos que reportam uma terapêutica não farmacológica insuficiente no que se refere a exercício físico em pessoas com diabetes, mostrando muito potencial de melhoria no seu controlo tensional. ${ }^{36}$ Seria então esperada uma relação direta com o controlo tensional em pessoas com diabetes. Uma possível explicação para este resultado poderá estar relacionada com o facto da escala utilizada medir além do nível de atividade física também a motivação para o aumentar o que poderá levar a alguma inércia terapêutica, específica em doentes com maior motivação, esperando-se mais tempo até intensificação da terapêutica farmacológica. De facto, os doentes mais ativos têm demonstrado nos estudos um controlo tensional com menos fármacos ${ }^{22}$ e a atividade física auto-referida e a motivação para a fazer poderão não ser equivalentes à efetiva prática de mais exercício. De qualquer forma, será uma questão pertinente a investigar, nomeadamente em Portugal, e levanta a pertinência de se validar uma escala de atividade física apenas para Portugal.

Neste estudo, o facto de fumar está associado de forma independente a menor controlo da TA. Num estudo na Alemanha obtiveram-se resultados semelhantes mas depois de ajustadas as variáveis de confundimento este perdeu a significância estatística. ${ }^{35}$ De forma contrária a este achado, num estudo português, a frequência de síndrome metabólico foi maior entre os não fumadores e ex-fumadores do que naqueles com hábitos tabágicos. ${ }^{4}$ Mais uma vez um fator de risco cardio-vascular importante ${ }^{58}$ esteve relacionado com a TA aumentada no nosso estudo.

Portanto, múltiplos fatores demográficos, hábitos e fatores de risco parecem influenciar/predizer o controlo tensional dos doentes com diabetes seguidos nos cuidados de saúde primários portugueses, explicando $43,7 \%$ da variação tensional. Sendo assim, estes podem ajudar o médico a escolher a população diabética na qual deve haver mais atenção ao risco cardiovascular por ter mais probabilidade de descontrolo tensional havendo vigilância mais apertada e de forma mais agressiva a estes parâmetros (fumadores, ativos, com obesidade abdominal, maior pressão de pulso, menos formação e com diabetes de maior evolução). Além disso, estes sub-grupos poderão ter a TA aumentada por algum mecanismo específico e isso poderá ser útil ser investigado, no sentido de melhorar o controlo tensional e diminuir a morbimortalidade cardiovascular e total associada à diabetes.

Uma das limitações deste estudo é que a amostra não foi totalmente aleatória pois os médicos de família que recolheram os dados foram voluntários e selecionados através das redes sociais, o que implica um viés de seleção. 
Ainda assim, os doentes com diabetes foram escolhidos de forma aleatória e a amostra apresentou uma distribuição pelo país semelhante à distribuição dos doentes com diabetes em Portugal. ${ }^{23}$ Os doentes estudados correspondiam a frequentadores das consultas de diabetes dos cuidados de saúde primários e não incluía doentes com diagnóstico de depressão, pelo que não se poderão generalizar estes dados para toda a população diabética de Portugal. Na amostra, 60,2\% dos doentes com diabetes eram do sexo masculino e em Portugal estes representam cerca de 58\% dos doentes com diabetes. Em termos de idades, tinham $66,12 \pm 10,47$ anos, sendo que em Portugal a prevalência de diabetes é maior no grupo 60 - 79 anos. ${ }^{23}$ Portanto, apesar destas limitações, poderemos dizer que a amostra é aproximadamente representativa dos doentes com diabetes em Portugal.

Além disso, estiveram envolvidos 65 investigadores, dos quais 41 devolveram dados de doentes recrutados. Isto poderá levar a uma forma heterogénea na medição de certas variáveis como a tensão arterial e perímetro abdominal (viés inter-observador). Isto tentou ser minimizado por reuniões presenciais feitas e pelas instruções detalhadas enviadas por escrito. Estes investigadores foram voluntários e não receberam qualquer compensação, pelo que teve de se limitar a escolha de variáveis recolhidas não tendo incluído outros fatores sociodemográficos como estado laboral e civil, fatores de risco como a dislipidémia, caracterização de hábitos etílicos e hábitos nutricionais, caracterização da hipertensão e sua duração, caracterização das complicações, polimedicação e co-morbilidades, fatores estes que poderiam influenciar a adesão à terapêutica e o controlo tensional.

Outra limitação deste estudo foi o facto de ser transversal, não fornecendo informação sobre relação temporal nem de causalidade. Ainda assim, faz um retrato de uma realidade do controlo tensional associado à diabetes nos cuidados de saúde primários portugueses.

\section{CONCLUSÃO}

As variáveis que mostraram influência no pior controlo tensional das pessoas com diabetes, de forma independente, foram a formação académica mais baixa, o maior perímetro abdominal, maior pressão de pulso, maior nível de atividade física, hábitos tabágicos e menor duração da diabetes. Sendo assim, foram identificadas características de sub-grupos de pessoas com diabetes em que será importante a equipa de saúde intervir de forma mais intensiva. Isto implicará uma especial atenção às pessoas com diabetes de diagnóstico recente, em que será importante haver um foco mais precoce no controlo da tensão arterial, apesar da tendência para focar no controlo glicémico. Estas conclusões reforçam a importância de controlar a tensão nas pessoas com diabetes que têm mais fatores de risco cardiovascular especialmente as que têm maior perímetro abdominal e que são fumadoras e realça a importância de valorizar a pressão de pulso como fator de risco associado. Não se deverão desvalorizar os valores tensionais elevados, apesar de uma boa adesão à atividade física, mas sim atuar de forma mais intensiva nesta população. Será também importante tentar perceber, nas pessoas com menor formação, qual a sua compreensão do risco cardiovascular, qual a importância dada à adesão à terapêutica e quais as dificuldades no controlo tensional nesta população e assim determinar qual a melhor forma de o ultrapassar.

Sugerimos estudos longitudinais e abrangentes no sentido de confirmar estes achados e identificar a população-alvo a ter mais atenção em termos de RCV para diminuir a morbimortalidade desta doença com impacto crescente em todo o mundo.

\section{AGRADECIMENTOS}

Os autores agradecem a todas as unidades de saúde e médicos de família que colaboraram no recrutamento e na recolha de dados: Dagmara Paiva, João Monteiro, Manuel Tinoco, Ângela Neves, Raquel Braga, Maria José Teixeira, Carla Costa, Carla Morna, Maria Margarida Moreira, José Rui Caetano, Francisco Fachado, Lina Costa, Daniela Neves, Mónica Granja, Gabriela Poças, Helena Oliveira, Paulo Sousa Neves, Severina Nicora, Dilermando Sobral, José Augusto Simões, Celina Pires Rosa, Raquel Ferreira, Pedro Salgado Gomes, Ana Sofia Tavares, Helena Carvalho, António Pereira, Miguel Pereira, Vasco Queiroz, Liliana Constantino, Joana Azeredo, Luísa Carvalho, Paula Cristina Silva, Joana Abreu, Maria de Lurdes Tavares Bello, Tatiana Petrachi, Maria José Verdasca, Maria do Rosário Gonçalves, Andreia Luís, Marta Marquês, Joana Luís, Ana Andreia Matos, Raquel Henriques, Jaime Brito da Torre, Cineyde Barros, Natalyia Barchuk, Nuno Jacinto, Laura Gonçalves, Hermínia Caeiro, Viktoriia Vinagre, Nelson Brito, Margarida Feteira, Rui Miranda, Susana Costa.

\section{PROTECÇÃO DE PESSOAS E ANIMAIS}

Os autores declaram que os procedimentos seguidos estavam de acordo com os regulamentos estabelecidos pelos responsáveis da Comissão de Investigação Clínica e Ética e de acordo com a Declaração de Helsínquia da Associação Médica Mundial.

\section{CONFIDENCIALIDADE DOS DADOS}

Os autores declaram ter seguido os protocolos do seu centro de trabalho acerca da publicação de dados.

\section{CONFLITOS DE INTERESSE}

Sem conflitos de interesse.

\section{FONTE DE FINANCIAMENTO}

Sem financiamentos externos, despesas pagas pelos investigadores. 


\section{REFERÊNCIAS}

1. International Diabetes Federation. IDF diabetes atlas [e-book]. $7^{\text {th }}$ ed. 2015. [consultado 2016 fev 22]. Disponível em: http://www.diabetesatlas. org/.

2. Observatório da Diabetes. Diabetes factos e números 2014 - relatório anual. [e-book] 2015. [consultado 2016 fev 22]. Disponível em https:// www.dgs.pt/estatisticas-de-saude/estatisticas-de-saude/publicacoes/ diabetes-factos-e-numeros-7-edicao.aspx.

3. American Diabetes Association. Cardiovascular disease and risk management. Diabetes Care. 2016;39:S60-71.

4. Fiuza M, Cortez-Dias N, Martins S, Belo A, VALSIM study investigators. Metabolic syndrome in Portugal: prevalence and implications for cardiovascular risk--results from the VALSIM Study. Rev Port Cardiol. 2008;27:1495-529.

5. Gæde $P$, Lund-Andersen $H$, Parving $H H$, Pedersen O. Effect of a multifactorial intervention on mortality in type 2 diabetes. N Engl J Med. 2008;358:580-91.

6. Kannel WB, McGee DL. Diabetes and cardiovascular risk factors: the Framingham study. Circulation. 1979;59:8-13.

7. Cortez-Dias N, Martins S, Belo A, Fiuza M, VALSIM. Prevalence, management and control of diabetes mellitus and associated risk factors in primary health care in Portugal. Rev Port Cardiol. 2010;29:509-37.

8. UK Prospective Diabetes Study Group. Tight blood pressure control and risk of macrovascular and microvascular complications in type 2 diabetes: UKPDS 38. BMJ. 1998;317:703-13.

9. The ADVANCE Collaborative Group. Intensive blood glucose control and vascular outcomes in patients with type 2 diabetes. $\mathrm{N}$ Engl J Med. 2008;358:2560-72.

10. The ACCORD Study Group. Effects of intensive blood-pressure control in type 2 diabetes mellitus. N Engl J Med. 2010;362:1575-85.

11. Hypertension in Diabetes Study Group. Hypertension in Diabetes Study (HDS): I. Prevalence of hypertension in newly presenting type 2 diabetic patients and the association with risk factors for cardiovascular and diabetic complications. J Hypertens. 1993;11:309-17.

12. UK Prospective Diabetes Study (UKPDS) Group. Effect of intensive blood-glucose control with metformin on complications in overweight patients with type 2 diabetes (UKPDS 34). Lancet. 1998;352:854-65.

13. Hemmingsen B, Lund SS, Gluud C, Vaag A, Almdal T, Wetterslev J. Targeting intensive glycaemic control versus targeting conventional glycaemic control for type 2 diabetes mellitus. In: The Cochrane Collaboration, ed. Cochrane Database of Systematic Reviews. Chichester: John Wiley \& Sons, Ltd; 2009.

14. UK Prospective Diabetes Study Group. Cost effectiveness analysis of improved blood pressure control in hypertensive patients with type 2 diabetes: UKPDS 40. BMJ. 1998;317:720-6.

15. McBrien K, Rabi DM, Campbell N, Barnieh L, Clement F, Hemmelgarn $\mathrm{BR}$, et al. Intensive and standard blood pressure targets in patients with type 2 diabetes mellitus: systematic review and meta-analysis. Arch Intern Med. 2012;172:1296-303.

16. Penno G, Solini A, Bonora E, Fondelli C, Orsi E, Zerbini G, et al. Gender differences in cardiovascular disease risk factors, treatments and complications in patients with type 2 diabetes: the RIACE Italian multicentre study. J Intern Med. 2013;274:176-91.

17. Duggirala MK, Cuddihy RM, Cuddihy MT,Naessens JM, Stephen SC, Jayawant NM, et al. Predictors of blood pressure control in patients with diabetes and hypertension seen in primary care clinics. Am J Hypertens. 2005;18:833-8.

18. Chew BH, Mastura I, Shariff-Ghazali S, Ping Yein L, Ai Teng C, Zaiton $A$, et al. Determinants of uncontrolled hypertension in adult type 2 diabetes mellitus: an analysis of the Malaysian diabetes registry 2009. Cardiovasc Diabetol. 2012;11:54.

19. Abougalambou SS, Abougalambou AS. A study evaluating prevalence of hypertension and risk factors affecting on blood pressure control among type 2 diabetes patients attending teaching hospital in Malaysia. Diabetes Metab Syndr Clin Res Rev. 2013;7:83-6.

20. Ahluwalia IB, Tessaro I, Greenlund KJ, Ford ES. Factors associated with control of hypertension, hypercholesterolemia, and diabetes among lowincome women in West Virginia. J Womens Health. 2010;19:417-24.

21. de Pablos-Velasco P, Gonzalez-Albarran O, Estopiñan V, Khanbhai A. Blood pressure, antihypertensive treatment and factors associated with good blood pressure control in hypertensive diabetics: the Tarmidas study. J Hum Hypertens. 2007;21:664-72.

22. Duclos M, Dejager S, Postel-Vinay N, di Nicola S, Quéré S, Fiquet B. Physical activity in patients with type 2 diabetes and hypertension - insights into motivations and barriers from the MOBILE study. Vasc
Health Risk Manag. 2015;11:361-71.

23. Gardete-Correia L, Boavida JM, Raposo JF, Mesquita AC, Fona C, Carvalho $\mathrm{R}$, et al. First diabetes prevalence study in Portugal: PREVADIAB study. Diabet Med. 2010;27:879-81.

24. Piepoli MF, Hoes AW, Agewall S, Christian A, Carlos B, Alberico LC, et al. 2016 European Guidelines on cardiovascular disease prevention in clinical practice. Eur Heart J. 2016;37:2315-81.

25. Mancia G, Fagard R, Narkiewicz K, Redon J, Zanchetti A. 2013 ESH/ ESC guidelines for the management of arterial hypertension. Eur Heart J. 2013;34:2159-219.

26. Núcleo de Exercício e Saúde, Faculdade de Motricidade Humana, Universidade Técnica de Lisboa, ed. Aconselhamento para a actividade física PACE. Oeiras: CM; 2003.

27. Delgado $A B$, Lima ML. Contributo para a validação concorrente de uma medida de adesão aos tratamentos. Psicol Saúde Amp Doenças. 2001;2:81-100.

28. de Pablos-Velasco P, Parhofer KG, Bradley C, Eveline E, Linda G, Pierre $\mathrm{M}$, et al. Current level of glycaemic control and its associated factors in patients with type 2 diabetes across Europe: data from the PANORAMA study. Clin Endocrinol. 2014;80:47-56.

29. Stone MA, Charpentier G, Doggen K, Kuss O, Lindblad U, Kellner C, et al. Quality of care of people with type 2 diabetes in eight european countries. Diabetes Care. 2013;36:2628-38.

30. Wong ND, Patao C, Wong K, Malik S, Franklin SS, Iloeje U. Trends in control of cardiovascular risk factors among US adults with type 2 diabetes from 1999 to 2010: Comparison by prevalent cardiovascular disease status. Diab Vasc Dis Res. 2013;10:505-13.

31. Rückert IM, Schunk M, Holle R, Schipf $S$, Volzke H, Kluttig A, et al. Blood pressure and lipid management fall far short in persons with type 2 diabetes: results from the DIAB-CORE Consortium including six German population-based studies. Cardiovasc Diabetol. 2012;11:50.

32. Kemp TM, Barr EL, Zimmet PZ, Cameron AJ, Welborn TA, Colagiuri S, et al. Glucose, lipid, and blood pressure control in Australian adults with type 2 diabetes. Diabetes Care. 2005;28:1490-92.

33. Bolen SD, Samuels TA, Yeh HC, Marinopoulos S, McGuire M, Abuid $M$, et al. Failure to intensify antihypertensive treatment by primary care providers: a cohort study in adults with diabetes mellitus and hypertension. J Gen Intern Med. 2008;23:543-50.

34. Grant RW, Cagliero E, Murphy-Sheehy P, Singer DE, Nathan DM, Meigs JB. Comparison of hyperglycemia, hypertension, and hypercholesterolemia management in patients with type 2 diabetes. Am J Med. 2002;112:603-9.

35. Rückert IM, Maier W, Mielck A, Schipf S, Volzke H, Kluttig A, et al. Personal attributes that influence the adequate management of hypertension and dyslipidemia in patients with type 2 diabetes. Results from the DIAB-CORE Cooperation. Cardiovasc Diabetol. 2012;11:120.

36. Owen A, Retegan C, Rockell M, Jennings G, Reid C. Inertia or inaction? blood pressure management and cardiovascular risk in diabetesł. Clin Exp Pharmacol Physiol. 2009;36:643-7.

37. Pinkhasov RM, Wong J, Kashanian J, Lee M, Samadi DB, Pinkhasov $\mathrm{MM}$, et al. Are men shortchanged on health? Perspective on health care utilization and health risk behavior in men and women in the United States. Int J Clin Pract. 2010;64:475-87.

38. Courtenay WH. Constructions of masculinity and their influence on men's well-being: a theory of gender and health. Soc Sci Med. 2000;50:1385401.

39. Benavente A, Rosa A, Costa AF da, Ávila P. A literacia em Portugal: resultados de uma pesquisa extensiva e monográfica. Fundação Calouste Gulbenkian, Conselho Nacional de Educação; [e-book] 1996. [consultado 2016 fev 22] Disponível em: http://www.cnedu.pt/ $\mathrm{pt} /$ publicacoes/estudos-e-relatorios/outros/799-a-literacia-em-portugalresultados-de-uma-pesquisa-extensiva-e-monografica.

40. Gomes MC, Ávila P, Sebastião J, Costa AF. Novas análises dos níveis de literacia em Portugal: comparações diacrónicas e internacionais. In: Actas Do IV Congresso Português de Sociologia. Sociedade Portuguesa: Passados Recentes. Coimbra: FEUC; 2000.

41. Walker RJ, Gebregziabher M, Martin-Harris B, Egede LE. Independent effects of socioeconomic and psychological social determinants of health on self-care and outcomes in type 2 diabetes. Gen Hosp Psychiatry. 2014;36:662-8.

42. Guillausseau P. Influence of oral antidiabetic drugs compliance on metabolic control in type 2 diabetes. A survey in general practice. Diabetes Metab. 2003;29:79-81.

43. Schillinger D, Grumbach K, Piette J, Wang F, Osmond D, Daher C, 
et al. Association of health literacy with diabetes outcomes. JAMA. 2002;288:475-82.

44. Ahmad NS, Islahudin F, Paraidathathu T. Factors associated with good glycemic control among patients with type 2 diabetes mellitus. J Diabetes Investig. 2014:5:563-9.

45. Benoit SR, Fleming R, Philis-Tsimikas A, Ji M. Predictors of glycemic control among patients with type 2 diabetes: A longitudinal study. BMC Public Health. 2005;5:36.

46. Al-Rasheedi AA. The role of educational level in glycemic control among patients with type II diabetes mellitus. Int J Health Sci. 2014;8:177-87.

47. Nemeh AA, Yousef SK, Aysha MA. Glycemic control and its determinants among patients with type 2 diabetes mellitus attending a teaching hospital. J Diabetes Metab. 2011;2:1-5.

48. Khattab M, Khader YS, Al-Khawaldeh A, Ajlouni K. Factors associated with poor glycemic control among patients with Type 2 diabetes. J Diabetes Complications. 2010;24:84-9.

49. Chan JC, Gagliardino JJ, Baik SH, Cantelot JM, Ferreira SR, Hancu $\mathrm{N}$, et al. Multifaceted determinants for achieving glycemic control. The International Diabetes Management Practice Study (IDMPS). Diabetes Care. 2009;32:227-33.

50. Harris SB, Ekoé JM, Zdanowicz Y, Webster-Bogaert S. Glycemic control and morbidity in the Canadian primary care setting (results of the diabetes in Canada evaluation study). Diabetes Res Clin Pract. 2005;70:90-7.

51. Qiao Q, Group TDS. Comparison of different definitions of the metabolic syndrome in relation to cardiovascular mortality in European men and women. Diabetologia. 2006;49:2837-46.

52. Ford ES. Risks for all-cause mortality, cardiovascular disease, and diabetes associated with the metabolic syndrome: a summary of the evidence. Diabetes Care. 2005;28:1769-78.

53. Balkau B, Deanfield JE, Després JP, Bassan JP, Fox KA, Smith SC, et al. International Day for the Evaluation of Abdominal Obesity (IDEA): a study of waist circumference, cardiovascular disease, and diabetes mellitus in 168,000 primary care patients in 63 countries. Circulation. 2007;116:1942-51.

54. Knudsen ST, Poulsen PL, Hansen KW, Ebbehøj E, Bek T, Mogensen $\mathrm{CE}$. Pulse pressure and diurnal blood pressure variation: association with micro- and macrovascular complications in type 2 diabetes. Am J Hypertens. 2002;15:244-50

55. Norris SL, Zhang X, Avenell A, Gregg E, Schmid CH, Lau J. Longterm non-pharmacological weight loss interventions for adults with prediabetes. In: Cochrane Database of Systematic Reviews. Chichester: John Wiley \& Sons, Ltd; 2005. [consultado 2016 fev 22] Disponível em: http://onlinelibrary.wiley.com/doi/10.1002/14651858.CD005270/ abstract.

56. Hayashino Y, Jackson JL, Fukumori N, Nakamura F, Fukuhara S. Effects of supervised exercise on lipid profiles and blood pressure control in people with type 2 diabetes mellitus: a meta-analysis of randomized controlled trials. Diabetes Res Clin Pract. 2012;98:349-60.

57. Figueira FR, Umpierre D, Cureau FV, Zucatti AT, Dalzochio MB, Leitão CB, et al. Association between physical activity advice only or structured exercise training with blood pressure levels in patients with type 2 diabetes: a systematic review and meta-analysis. Sports Med. 2014;44:1557-72

58. Hughes JR. A quantitative estimate of the clinical significance of treating tobacco dependence. Am J Prev Med. 2010;39:285-6. 